\title{
Correction to: The Casting Powders Book
}

\section{Correction to: \\ K.C. Mills and C.-Å. Däcker, The Casting Powders Book, https://doi.org/10.1007/978-3-319-53616-3}

Within this book, minor changes to some of the figure permissions and the readability of the chapters have been made and several figures have been replaced with new versions.

The updated version of the book can be found at https://doi.org/10.1007/978-3-319-53616-3 